\title{
Cosmological constraints with GRBs: homogeneous medium vs. wind density profile
}

\author{
G. Ghirlanda ${ }^{1}$, G. Ghisellini ${ }^{1}$, C. Firmani ${ }^{1,3}$, L. Nava $^{1,2}$, F. Tavecchio ${ }^{1}$, and D. Lazzati ${ }^{4}$ \\ 1 Osservatorio Astronomico di Brera, via Bianchi 46, Merate, Italy \\ e-mail: ghirlanda@merate.mi.astro.it \\ 2 Univ. di Milano-Bicocca, P. za della Scienza 3, 20126 Milano, Italy \\ 3 Instituto de Astronomía, UNAM, AP 70-264, 04510 México, DF, México \\ 4 JILA, Campus Box 440, University of Colorado, Boulder, C0 80309-440, USA
}

Received 18 November 2005 / Accepted 3 March 2006

\section{ABSTRACT}

\begin{abstract}
We present the constraints on the cosmological parameters obtained with the $E_{\text {peak }}-E_{\gamma}$ correlation found with the most recent sample of 19 GRBs with spectroscopically measured redshift and well determined prompt emission spectral and afterglow parameters. We compare our results obtained in the two possible uniform jet scenarios, i.e. assuming a homogeneous density profile (HM) or a wind density profile (WM) for the circumburst medium. We explore the improvements to the constraints of the cosmological parameters that could be reached with a large sample, $\sim 150 \mathrm{GRBs}$, in the future. Finally, we study the possibility to calibrate the slope of these correlations. Our optimization analysis suggests that $\sim 12 \mathrm{GRBs}$ with redshift $z \in(0.9,1.1)$ can be used to calibrate the $E_{\text {peak }}-E_{\gamma}$ with a precision better than $1 \%$. The same precision is expected for the same number of bursts with $z \in(0.45,0.75)$. This result suggests that we do not necessarily need a large sample of low $z$ GRBs for calibrating the slope of these correlations.
\end{abstract}

Key words. gamma rays: bursts - radiation mechanisms: non-thermal - X-rays: general - cosmology: miscellaneous

\section{Introduction}

Gamma Ray Bursts (GRBs) are presently detected out to very high redshifts (the new limit being GRB 050904 at $z=6.29$, Kawai et al. 2005) and this makes them extremely attractive for observational cosmology. They might have profound impact on (i) the study of the epoch of reionization; (ii) the characterization of the properties of the cosmic intergalactic medium; (iii) the description of the geometry of the Universe; and (vi) the investigation of the nature of Dark Energy. GRBs have recently been discussed (Ghirlanda et al. 2004a; Firmani et al. 2005; Lamb et al. 2005) as a possible new class of "standard candles" to be used to constrain the Universe models (Ghirlanda et al. 2004a; Firmani et al. 2005; Liang \& Zhang 2005). What "standardize" the GRB energetics is a tight relation between their rest frame collimation corrected energy $E_{\gamma}$ and the peak energy $E_{\text {peak }}$ of their $v F_{v}$ prompt emission spectra (Ghirlanda et al. 2004b). In the "standard" GRB scenario the collimation corrected energy is defined as $E_{\gamma}=E_{\gamma \text {,iso }}\left(1-\cos \theta_{\mathrm{j}}\right)$, where $E_{\gamma \text {,iso }}$ is the isotropic equivalent energy and $\theta_{\mathrm{j}}$ is the jet opening angle. This parameter can be derived from $t_{\mathrm{j}}$, i.e. the time when the afterglow light curve steepens, in two different scenarios: (a) assuming that the circumburst medium is homogeneous (HM) or (b) assuming a stratified density profile produced, for instance, by the wind of the GRB progenitor (WM). In both cases the jet is assumed to be uniform. Originally the $E_{\text {peak }}-E_{\gamma}$ correlation was derived in the HM case with 15 GRBs (Ghirlanda et al. 2004b) and resulted in a small scattered correlation $\left(E_{\text {peak }} \propto E_{\gamma}^{0.7}\right.$ with $\sigma \sim 0.15)$ which was used to derive interesting (though shallow) constraints on the cosmological parameters $\left(\Omega_{\mathrm{M}}, \Omega_{\Lambda}\right)$ and on the equation of state parameters of the Dark Energy (Firmani et al. 2005). Recently Nava et al. 2006 (N05) derived the
$E_{\text {peak }}-E_{\gamma}$ correlation in the WM case. This correlation is less scattered, i.e. $\sigma=0.08$, than in the HM case and it is linear, i.e. $E_{\text {peak }} \propto E_{\gamma}$. N05 discussed its implications for the understanding of the dynamics and radiative processes of GRBs. Although the $E_{\text {peak }}-E_{\gamma}$ correlations (both in the HM and WM case) are model dependent, their consistency with the completely empirical correlation between $E_{\text {peak }}, E_{\gamma}$ and $t_{\mathrm{j}}$ found by Liang \& Zhang (2005 - hereafter LZ05), suggests that the model parameters are not much dispersed. It is therefore worth to derive the cosmological constraints with the Ghirlanda correlation in both the HM and WM scenario and compare the results. In order to use GRBs as cosmological tools, through the above correlations, three foundamental parameters, $E_{\text {peak }}, E_{\gamma}$ and $t_{\text {jet }}$, should be accurately measured or inferred. This requirement also applies to the empirical correlation of LZ05. Therefore only a limited number of GRBs, i.e. 19 out of $\sim 70$ (up to Nov. 2005) with measured $z$, can be used as standard candles. It also appears that the limited energy range of BAT onboard Swift (15-150 keV) allows to constrain only with moderate accuracy the $E_{\text {peak }}$ of particularly bright-soft bursts. However, given the perspective of the cosmological investigation through GRBs, it is worth exploring the power of using GRBs as cosmological probes. Another still open issue related to the use of GRBs as standard candles is the so called "circularity problem" (see Ghisellini et al. 2005). This is due to the fact that the small number of GRBs with spectroscopic measured redshifts and their wide dispersion in $z$ does not allow to calibrate the Ghirlanda correlation, which, in turn, depends on the cosmological parameters that we want to constrain. To the aim of calibrating this spectral-energy correlation, GRBs at low redshift are required. In fact for $z<0.1$ the difference in the luminosity distance computed for different choices of the cosmological models [for $\Omega_{\mathrm{M}}, \Omega_{\Lambda} \in(0,1)$ ] is less than $8 \%$. 
However, if (long) GRBs are produced by the death of massive stars, they should roughly follow the cosmic star formation history (SFR) and we should expect that the rate of low redshift events is quite small at $z<0.1$. Instead, a considerably large number of GRBs with $z>1$ should be collected in the next years by presently flying instruments (Swift and Hete-II). At such large redshifts, the cosmological models starts to play an important role. However, if it will be possible to have a sufficient number of GRBs with a similar redshift, it might still be possible to calibrate the slope of these correlations with high redshift GRBs.

The aim of this work is to find the cosmological constraints by using the $E_{\text {peak }}-E_{\gamma}$ correlation in the HM and WM case (Sect. 3) and to describe the level of precision that could be achieved with a large population of bursts (Sect. 4). Finally, we study the possibility to calibrate the slope of these relations with GRB samples at intermediate redshifts (Sect. 5).

\section{The $E_{\text {peak }}-E_{\gamma}$ correlation in the $\mathrm{HM}$ and $\mathrm{WM}$ case}

The jet opening angle $\theta_{\mathrm{j}}$ in the two HM or WM density profile scenarios is:

$$
\begin{aligned}
\theta_{\mathrm{j}} & =0.161\left(\frac{t_{\mathrm{j}, \mathrm{d}}}{1+z}\right)^{3 / 8}\left(\frac{n \eta_{\gamma}}{E_{\gamma, \mathrm{iso}, 52}}\right)^{1 / 8} \mathrm{HM} \\
\theta_{\mathrm{j}, \mathrm{w}} & =0.2016\left(\frac{t_{\mathrm{j}, \mathrm{d}}}{1+z}\right)^{1 / 4}\left(\frac{\eta_{\gamma} A_{*}}{E_{\gamma, \text { iso }, 52}}\right)^{1 / 4} \mathrm{WM}
\end{aligned}
$$

where $n$ is the constant circumburst density in the HM case (Sari et al. 1999) and $A_{*}$ is the normalization of the density profile $n(r)=5 \times 10^{11} A_{*} r^{-2} \mathrm{~g} \mathrm{~cm}^{-3}$ in the WM case (Chevalier \& $\mathrm{Li}$ 2000).

N05 derived the $E_{\mathrm{peak}}-E_{\gamma}$ correlation in the HM and WM case with 18 GRBs (sample updated to Sept. 2005) for which secure measurements of $z, E_{\text {peak }}$ and $t_{\mathrm{j}}$ have been reported in the literature. While completing this work the Hete-II and KonusWind satellites detected a new GRB (051022 - Olive et al. 2005) for which all the observables (i.e. $z=0.8$, Butler et al. 2005, $\left.E_{\text {peak }}, t_{\mathrm{j}}\right)$ required to compute the $E_{\text {peak }}-E_{\gamma}$ correlation were measured. We therefore added this burst to the sample of 18 events of N05. The spectrum of GRB 051022 as observed in the widest energy range by Konus-Wind (Golenetskii et al. 2005) is fitted by a powerlaw model with an high energy exponential cutoff: the low energy photon spectral index is $\alpha=-1.17 \pm 0.04$ and the $\left(E F_{\mathrm{E}}\right)$ peak energy is $E_{\text {peak }}=510 \pm 35 \mathrm{keV}$. The $2 \mathrm{keV}-20 \mathrm{MeV}$ energy fluence is $2.61 \pm 0.08 \times 10^{-4} \mathrm{erg} / \mathrm{cm}^{2}$. The analysis of the X-ray light curve has shown the presence of a possible jet break at $t_{\mathrm{b}}=2.9 \pm 0.2$ days (Racusin et al. 2005). For a standard cosmology with $\Omega_{\mathrm{M}}=0.3$ and $\Omega_{\Lambda}=h=0.7$ (and assuming typical values for the density and the wind profile normalization - see N05), we derive $\theta_{\mathrm{j}}(\mathrm{HM})=6.37 \pm 0.75^{\circ}$ and $\theta_{\mathrm{j}}(\mathrm{WM})=3.3 \pm 0.1^{\circ}$ for the HM and WM case. With the addition of this new bursts to the sample of 18 events of N05, we find $E_{\text {peak }} \propto E_{\gamma}^{0.67 \pm 0.04}$ (with a reduced $\chi_{\text {red }}^{2}=1.4$ and a Gaussian scatter of the 19 GRBs around this correlation with a $\sigma=0.1$ ) and $E_{\text {peak }} \propto E_{\gamma}^{1.00 \pm 0.06}$ (with a reduced $\chi_{\text {red }}^{2}=1.14$ and a Gaussian scatter of the 19 GRBs around this correlation with a $\sigma=0.08$ ) in the HM and WM case, respectively.

\section{Cosmological constraints}

To the aim of constraining the cosmological parameters $\Omega_{\mathrm{M}}$ and $\Omega_{\Lambda}$ we use the $E_{\text {peak }}-E_{\gamma}$ correlations following the Bayesian

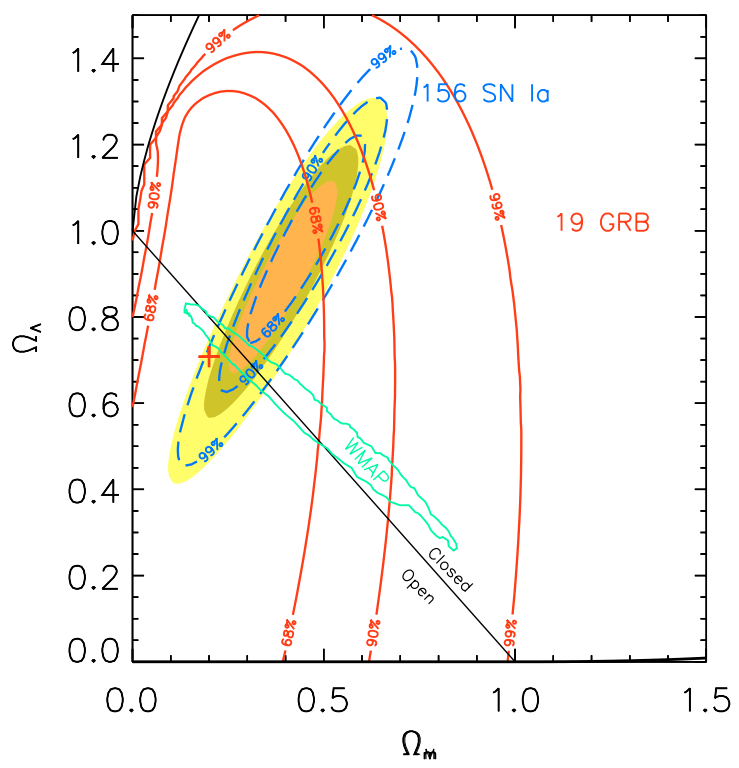

Fig. 1. Constraints on the cosmological parameters $\Omega_{\mathrm{M}}, \Omega_{\Lambda}$ obtained with the updated sample of 19 GRBs presented in Table 1 of N05 (to which GRB 051022 was added) in the homogeneous density case (HM). The solid (red) contours, obtained with the 19 GRBs alone, represent the $68.3 \%, 90 \%$ and $99 \%$ confidence regions. The center of these contours (red cross) corresponds to a minimum $\chi^{2}=15.25 / 17$ dof and has $\Omega_{\mathrm{M}}=0.23$ and $\Omega_{\Lambda}=0.81$. The contours obtained with the $156 \mathrm{SN}$ Ia of the "Gold" sample of Riess et al. (2004) are shown by the dashed (blue) lines. The joint GRB+SN constraints are represented by the shaded contours. We also show the $90 \%$ confidence contours obtained with the WMAP data (from Spergel et al. 2003).

method proposed by Firmani et al. (2005). The power of this optimized method is to circumvent the "circularity problem" arising from the fact that these correlations cannot be calibrated with the present sample of GRBs (see also Sect. 4).

\subsection{Homogeneous density}

First we assume a HM and, through Eq. (1), derive the cosmological constraints reported in Fig. 1. The results, obtained with the present sample of 19 GRBs, are fully consistent with those obtained by Firmani et al. (2005) with the smaller (and slightly different) sample of 15 events (Table 1 of GGL04). The slightly larger contours reported in Fig. 1 are due to the changes of the parameters (and associated uncertainties) for GRB 991216, 011211, 020124, 020405, 020813, 030226, 030328, 030329, 030429 present in both samples (see N05 for a detailed discussion). The joint GRB+SN fit (shaded regions in Fig. 1) is dominated by the small contours determined with the $156 \mathrm{SN}$ Ia of the "Gold" sample of Riess et al. (2004). However, we note that the GRB+SN Ia joint fit is consistent at the $68 \%$ confidence level with the concordance model $\Omega_{\mathrm{M}}, \Omega_{\Lambda}=(0.3,0.7)$.

\subsection{Wind density profile}

In the case of a wind density profile we can still derive the constraints on the cosmological parameters adopting Eq. (1) to compute the jet opening angle. We make the simplest assumption of $A_{*} \simeq 1$. This choice corresponds to assuming a typical mass loss rate $\dot{M}_{\mathrm{w}}=10^{-5} M_{\odot} \mathrm{yr}^{-1}$ and wind velocity $v_{\mathrm{w}}=10^{3} \mathrm{~km} \mathrm{~s}^{-1}$. We do not have any knowledge of the uncertainty associated to the parameter $A_{*}$. Fits of few afterglow light curves (Panaitescu \& Kumar 2000; Panaitescu 2005) showed typical values in the 


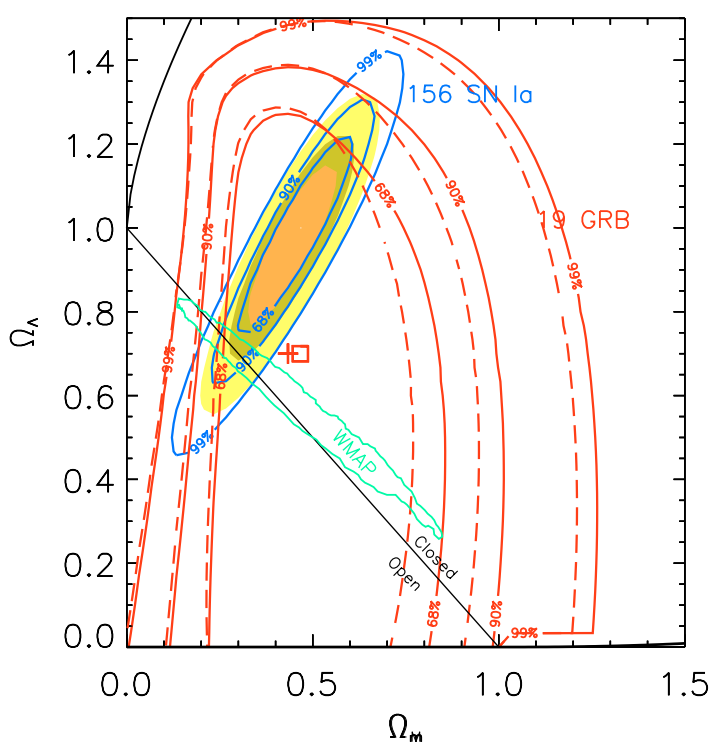

Fig. 2. Constraints on the cosmological parameters $\Omega_{\mathrm{M}}, \Omega_{\Lambda}$ obtained with the updated sample of 19 GRBs presented in Table 1 of N05 (to which GRB 051022 was added) in the wind density profile case (WM). The solid (red) contours, obtained with the 19 GRBs alone, represent the $68.3 \%, 90 \%$ and $99 \%$ confidence regions on the pair of cosmological parameters. The contours obtained with the 156 SN Ia of the "Gold" sample of Riess et al. 2004 are shown by the long-dashed (blue) lines. The joint GRB+SN constraints are represented by the shaded contours. We also report (dashed-red contours) the constraints obtained by assuming a fixed (i.e. cosmology invariant) linear correlation $E_{\text {peak }} \propto E_{\gamma}$.

range 0.2 and 2. In the HM case (see also Ghirlanda et al. 2004a) it is the density parameter $n$ to be characterized by a large uncertainty. In that case we assumed a factor 10 of uncertainty on this parameter. Therefore, we assumed the same uncertainty on the parameters $n$ and $A_{*}$ to homogeneously compare the cosmological constraints obtained in the HM and WM case. Figure 2 reports the results obtained in the WM case by assuming that $A_{*}$ can vary between 0.2 and 2 . The large uncertainty associated to this parameter makes the conotours slightly larger than those obtained in the HM case (see Fig. 1).

However the uncertainty on the $A_{*}$ parameter is completely unknown. The error on this parameter is propagated through Eq. (1) on $E_{\gamma}$. If we consider the concordance model and we fit the $E_{\text {peak }}-E_{\gamma}$ correlation by assuming a factor 10 of uncertainty, we find that its reduced $\chi^{2} \sim 4.8 / 17$ (d.o.f.), i.e. it is dominated by the statistical errors. Instead, as also discussed in Nava et al. (2006), we estimate that the minimal uncertainty on $A_{*}$ not to overestimate the statistical errors is $\sim 20 \%$. For this reason we also computed the constraints (Fig. 3) on the cosmological parameters by assuming such a minimal uncertainty on $A_{*}$.

In the WM case the $E_{\text {peak }}-E_{\gamma}$ correlation is linear (in the standard cosmology), and thus it has the remarkable property to be "Lorentz invariant", since both the $E_{\gamma}$ and $E_{\text {peak }}$ are boosted by $\sim 2 \Gamma$ when transforming from the comoving to the observer frame if we assume to view a uniform jet within the cone defined by its aperture angle. This property makes this correlation easier to be interpreted theoretically (see N05). We have then repeated our calculation assuming that the correlation remains linear in any cosmology. As discussed in N05, the linear Ghirlanda correlation obtained in the wind case is still consistent with the empirical correlation found by LZ05 and might have important implication for the physical interpretations of GRBs. If we then

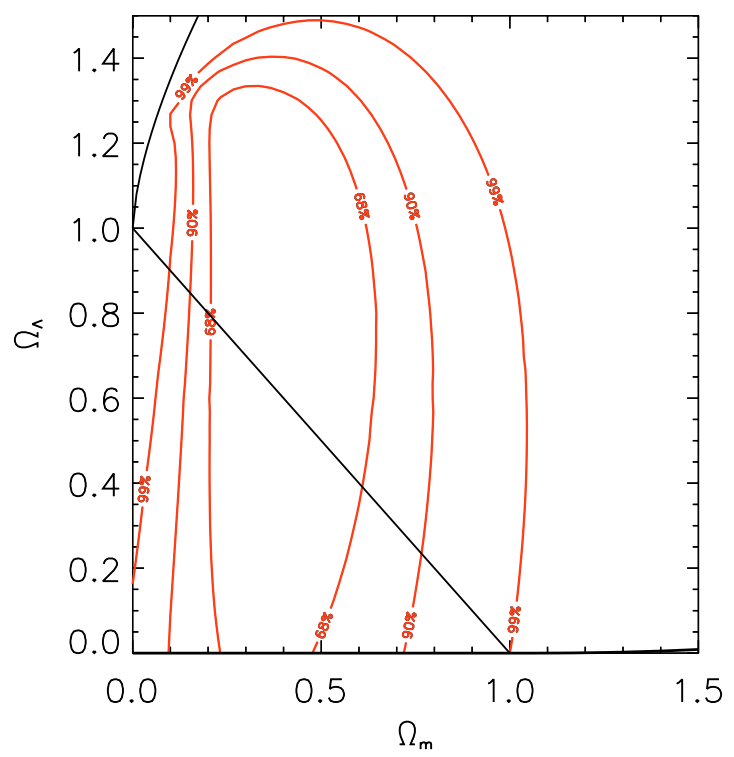

Fig. 3. Constraints on the cosmological parameters $\Omega_{\mathrm{M}}, \Omega_{\Lambda}$ obtained with the updated sample of 19 GRBs presented in Table 1 of N05 (to which GRB 051022 was added) in the wind density profile case (WM). The contours represent the $68.3 \%, 90 \%$ and $99 \%$ confidence regions on the pair of cosmological parameters. The contraints are obtained assuming a $20 \%$ uncertainty on the $A_{*}$ parameter. This uncertainty ensures that the fit of the $E_{\mathrm{peak}}-E_{\gamma}$ correlation is not dominated by the statistical errors.

fix the slope to 1 , the only free parameter remaining is the normalization. In this case we can find even better constraints (dotted red contours in Fig. 2 with respect to the case in which the correlation is left free to vary in every cosmology).

\section{GRB sample simulation}

In order to fully appreciate the potential use of GRBs for the cosmological investigation, we simulate a sample of bursts comparable in number to the "Gold" sample of 156 SN Ia. Similar simulations have been presented in the literature (Xu et al. 2005; Liang \& Zhang 2005); however different a-priori assumptions can be made on the properties of the simulated sample and the results are clearly dependent on these assumptions. In particular, simulations based on the observed parameters (Xu et al. 2005) strongly depend on the selection effects on these quantities.

We adopt here a method which makes use of the intrinsic properties of GRBs as described by the Ghirlanda and the Amati correlations (Amati et al. 2002). We use the most updated version of these correlations (N05) as found with the sample of 19 GRBs. With this sample the Amati correlation results:

$\left(\frac{E_{\mathrm{p}}^{\prime}}{100 \mathrm{keV}}\right)=(0.361 \pm 0.02)\left(\frac{E_{\gamma, \text { iso }}}{7.6 \times 10^{52} \mathrm{erg}}\right)^{0.57 \pm 0.02}$

with a reduced $\chi_{\text {red }}^{2}=5.22$ for 16 d.o.f.

The assumptions of our simulated GRB sample are:

- we assume that GRBs have an "isotropic energy" function which is described by a powerlaw $N\left(E_{\text {iso }}\right) \propto E_{\text {iso }}^{\delta}$ for $E_{\text {iso,min }}<E_{\text {iso }}<E_{\text {iso,max }}$ (e.g. Firmani et al. 2004). Further we assume that GRBs follow the cosmic star formation rate (SFR);

- we use the Amati correlation (Eq. (2)) to derive the peak energy $E_{\text {peak }}$; 

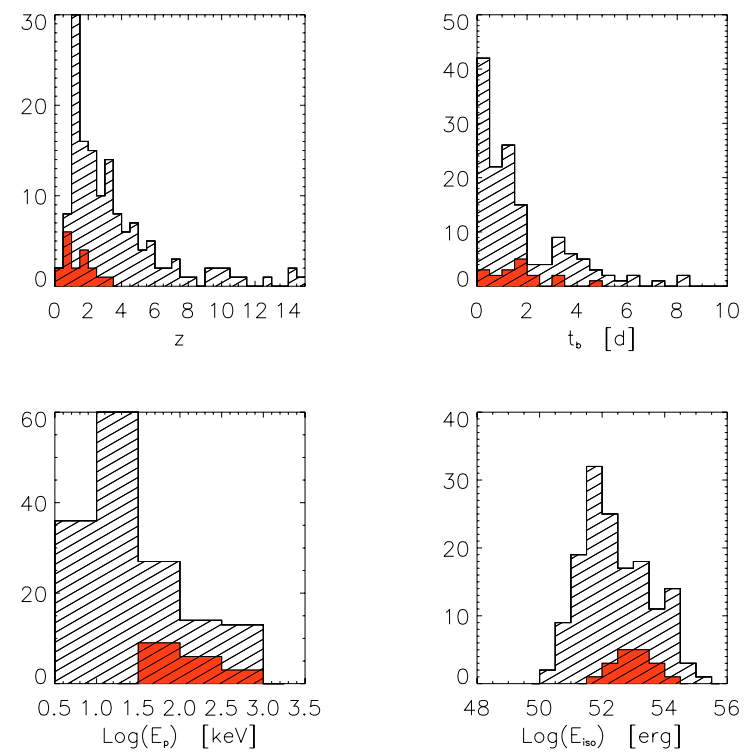

Fig. 4. Distributions of redshift $z$, jet break time $t_{\mathrm{b}}$, observed peak energy $E_{\text {peak }}^{\text {obs }}$ and isotropic equivalent energy $E_{\text {iso }}$ for the 150 simulated bursts (hatched histograms). Also show (solid filled histogram) are the distributions of the sample of 18 GRBs of N05 used to derive the $E_{\text {peak }}-E_{\text {iso }}$ correlation.

- we model the scatter of the simulated GRBs around the Amati correlation with a Gaussian distribution with $\sigma=0.3$ (which corresponds to the present scatter of the 19 GRBs around their best fit correlation - Eq. (2));

- we use the Ghirlanda correlation as found with the 19 GRBs (Eq. (1)) to calculate $E_{\gamma}$;

- we model the scatter of the simulated GRBs around the Ghirlanda correlation with a Gaussian distribution with $\sigma=$ 0.1 ( 0.08 for the wind case - see N05);

- we derive the jet opening angle $\theta_{\mathrm{j}}$ and the corresponding jet break time $t_{\mathrm{j}}$;

- we assume that the simulated GRB spectra are described by a Band model spectrum (Band et al. 1993) with typical low and high energy spectral photon indices $\alpha=-1.0$ and $\beta=-2.5$ and require that the simulated GRB fluence in the $2-400 \mathrm{keV}$ energy band is above a typical instrumental detection threshold of $\sim 10^{-7} \mathrm{erg} / \mathrm{cm}^{2}$. This corresponds roughly to the present threshold of Hete-II in the same energy band.

Following the procedure described above we built a sample of 150 GRBs with the relevant parameters: $z, E_{\mathrm{iso}}, E_{\mathrm{p}}, t_{\mathrm{j}, \mathrm{j}}$. The errors associated to these parameters are assumed to be cosmology invariant and they are set to $10 \%, 20 \%$ and $20 \%$ for $E_{\text {iso }}, E_{\mathrm{p}}$, $t_{\text {, j }}$ respectively. For our simulation we considered the Ghirlanda correlation in the WM case and adopted the SFR\#2 of Porciani \& Madau (2001). We modeled the GRB intrinsic isotropic energy function with a powerlaw with $\delta=-1.3$ between two limiting energies $\left(10^{49}-10^{55} \mathrm{erg}\right)$. This particular choice of parameters is due to the requirement that the distributions of the relevant quantities (shown in Fig. 4) of the simulated sample are consistent with the same distributions for the present sample of 19 GRBs. The sample is simulated in the standard cosmology $\left(\Omega_{\mathrm{M}}=0.3\right.$ and $\left.\Omega_{\Lambda}=h=0.7\right)$. We show the distribution of $z, t_{\mathrm{b}}, E_{\mathrm{p}}$ and $E_{\text {iso }}$ for the 150 simulated bursts in comparison with the same distributions of the 19 GRBs in Fig. 4. We also note that by choosing a steeper energy function we obtain a much larger number of XRF and XRR with respect to normal GRBs.

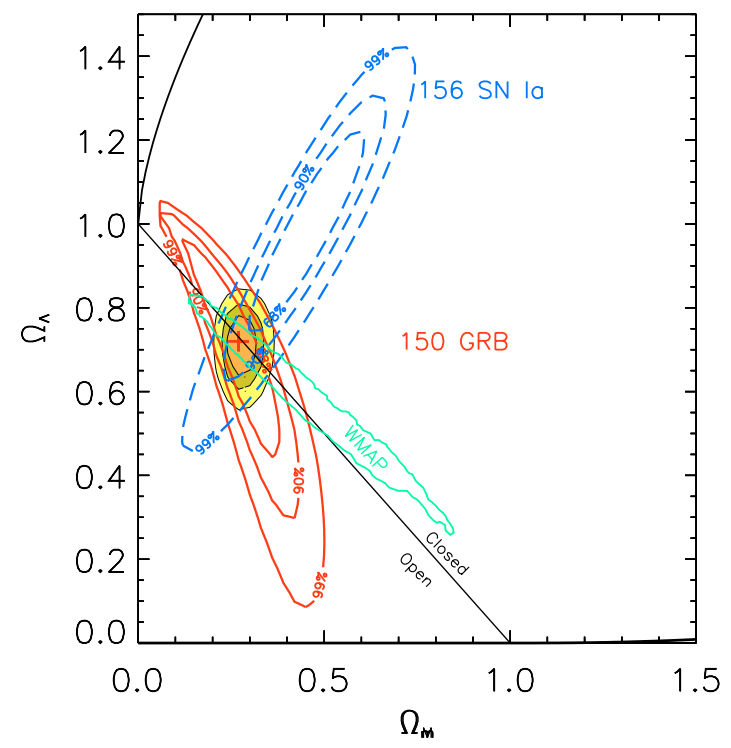

Fig. 5. Wind density profile case. Constraints on the cosmological parameters $\Omega_{\mathrm{M}}, \Omega_{\Lambda}$ obtained with the simulated sample of 150 GRBs. The solid (red) contours, obtained with the 19 GRBs alone, represent the $68.3 \%, 90 \%$ and $99 \%$ confidence regions on the pair of cosmological parameters. The contours obtained with the 156 SN Ia of the "Gold" sample of Riess et al. (2004) are shown by the dashed (blue) lines. The joint GRB+SN constraints are represented by the shaded contours.

The results obtained with the sample of 150 simulated GRBs is presented in Fig. 5: in this case the constraints are comparable with those obtained with SN Ia. The minimum of the GRB contours (cross in Fig. 5) corresponds to $\Omega_{\mathrm{M}}=0.27$ and $\Omega_{\Lambda}=0.72$. By comparing the $1 \sigma$ contours of GRB alone from Fig. 5 to the same contours (solid line) of Fig. 2 (obtained with the 19 GRBs), we note that there is an improvement (of roughly a factor 10) with the sample of 150 bursts.

It is evident the different orientation of the GRB contours (see Ghisellini et al. 2005) with respect to SN Ia due to the "topology" of the luminosity distance as a function of the $\Omega_{\mathrm{M}}-\Omega_{\Lambda}$ parameters. Most GRBs of our simulated sample are at $z \sim 2$, and this explains the tilt of the contours obtained in the $\Omega$ plane (Ghisellini et al. 2005). Clearly the simulated sample depends on the assumptions: in particular we have no knowledge of the burst intrinsic energy function $N\left(E_{\gamma, \text { iso }}\right)$. However, if we accept the hypothesis to model it with a simple powerlaw, we can change the slope and also include the effect of the redshift evolution. We tested the dependence of these assumptions on the constraints reported in Fig. 5 and found that the major effect of assuming different $\delta$ values and a $(1+z)$ evolutionary factor is to change the redshift distribution of the simulated sample and therefore to change the orientation of the GRB contours in Fig. 5. The same happens if we adopt, for the same choice of parameters reported above, a different SFR.

Further, we can also use the CMB priors. First we assume the cosmological constant model with the $2 \mathrm{CMB}$ priors, i.e. (i) $\Omega_{\mathrm{tot}}=1$ and (ii) $\Omega_{\mathrm{M}}=0.14 / h^{2}$. In this case the only free parameter is $h$ (or equivalently $\Omega_{\mathrm{M}}$ ). We obtain the best fit values of $\Omega_{\mathrm{M}}=0.27 \pm 0.02$ and $\Omega_{\Lambda}=0.73 \pm 0.02$.

\subsection{Dark energy EOS}

One of the major promises of the cosmological use of GRBs is related to the possibility to study the nature of Dark Energy with such a class of "standard candles" extending out to very large 


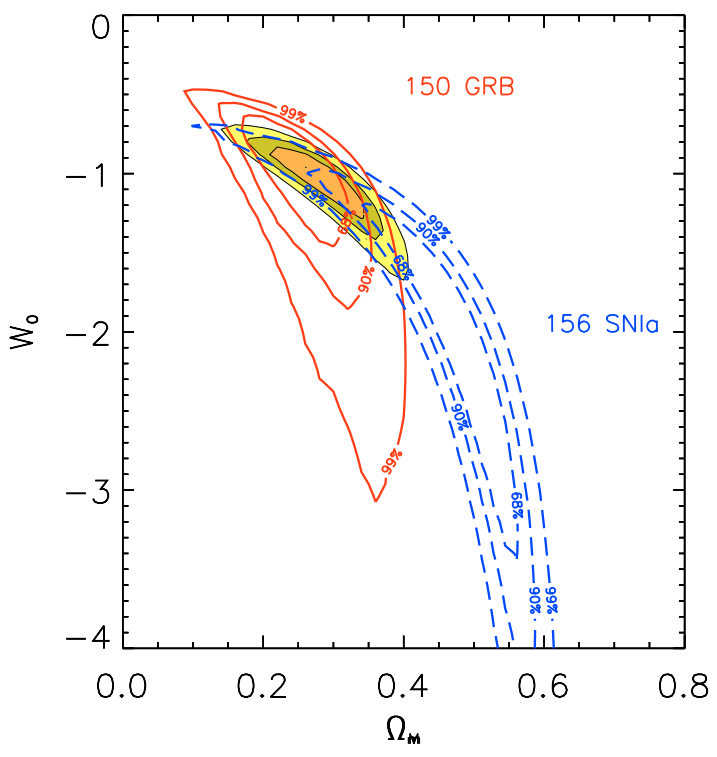

Fig. 6. Constraints on the cosmological parameters $w_{0}, \Omega_{\mathrm{M}}$ obtained with the 150 simulted GRBs (red contours) compared with the same contours obtained with the 156 SNIa of the "Gold" sample. A flat universe is assumed $\left(\Omega_{\text {tot }}=1\right)$.

redshifts. With the present sample of 19 GRBs we can explore the equation of state (EOS) of DE, which can be parametrized in different ways. Given the already considerably large dispersion of GRB redshifts (i.e. between 0.168 to 3.2 for the 19 GRBs of our sample) we adopt the parametrization proposed by Linder $\&$ Huterer (2005) for the EOS of DE, i.e. $P=w(z) \rho$, where:

$w(z)=w_{0}+\frac{w_{\mathrm{a}} z}{1+z}$

With this assumption the luminosity distance, as derived from the Friedmann equations, is

$$
\begin{aligned}
d_{\mathrm{L}}\left(z ; \Omega_{\mathrm{M}},\right. & \left.w_{0}, w_{\mathrm{a}}\right)=\frac{c(1+z)}{H_{0}} \int_{0}^{z} \mathrm{~d} z\left[\Omega_{\mathrm{M}}(1+z)^{3}\right. \\
& \left.+\left(1-\Omega_{\mathrm{M}}\right)(1+z)^{3+3 w_{0}+3 w_{\mathrm{a}}} \exp \left(-3 w_{\mathrm{a}} \frac{z}{1+z}\right)\right]^{-1 / 2}
\end{aligned}
$$

which depends on the $\left(w_{0}, w_{\mathrm{a}}\right)$ parameters. Note that Eq. (4) is derived with the prior of a flat Universe.

First we can assume the CMB prior of a flat universe together with the assumption of a non-evolving equation of state of the Dark Energy (i.e. $w_{\mathrm{a}}=0$ ). We show in Fig. 6 the contours obtained with the sample of 150 simulated GRBs and compare with the same constraints derived with the 156 SN Ia of the "Gold" sample. The constraints on $w_{\mathrm{a}}, w_{0}$ are reported in Fig. 7, assuming $\Omega_{\mathrm{M}}=0.3$.

\section{The calibration of the spectral energy correlations}

The cosmological use of the $E_{\text {peak }}=K \cdot E_{\gamma}^{g}$ correlation suffers from the so called "circularity problem" (Ghirlanda et al. 2004a; Ghisellini et al. 2005): this means that both the slope $g$ and the normalization $K$ of the correlation are cosmology dependent. In fact, of the two rest frame quantities $E_{\text {peak }}$ and $E_{\gamma}$ that are used to compute the Ghirlanda correlation, the second one $\left(E_{\gamma}\right)$ depends on the cosmological model through the luminosity distance $d_{\mathrm{L}}(z ; \Omega)$.

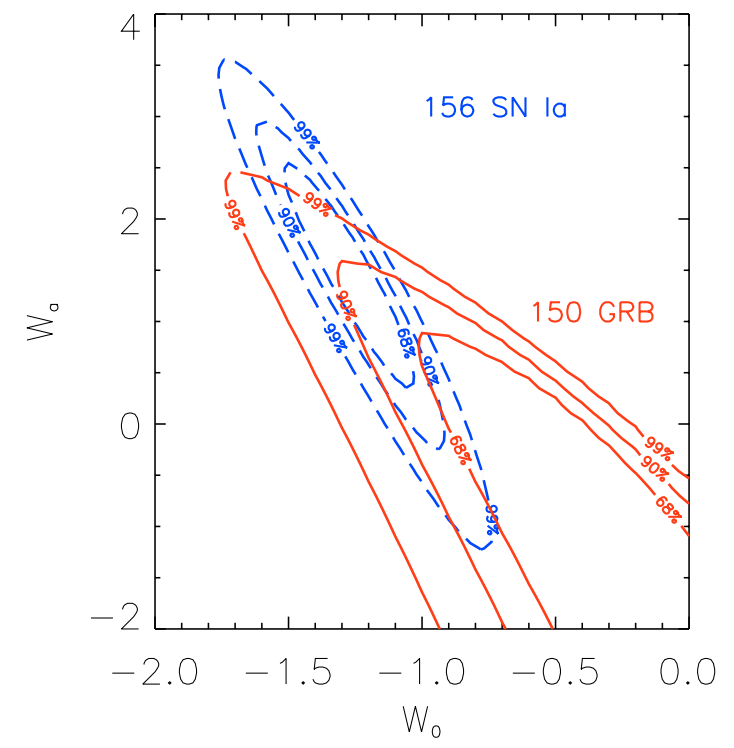

Fig. 7. Constraints on the cosmological parameters $w_{0}, w_{\text {a }}$ obtained with the 150 simulted GRBs (red contours) compared with the same contours obtained with the 156 SNIa of the "Gold" sample. A flat universe $\left(\Omega_{\text {tot }}=1\right)$ with $\Omega_{\mathrm{M}}=0.3$ is assumed.

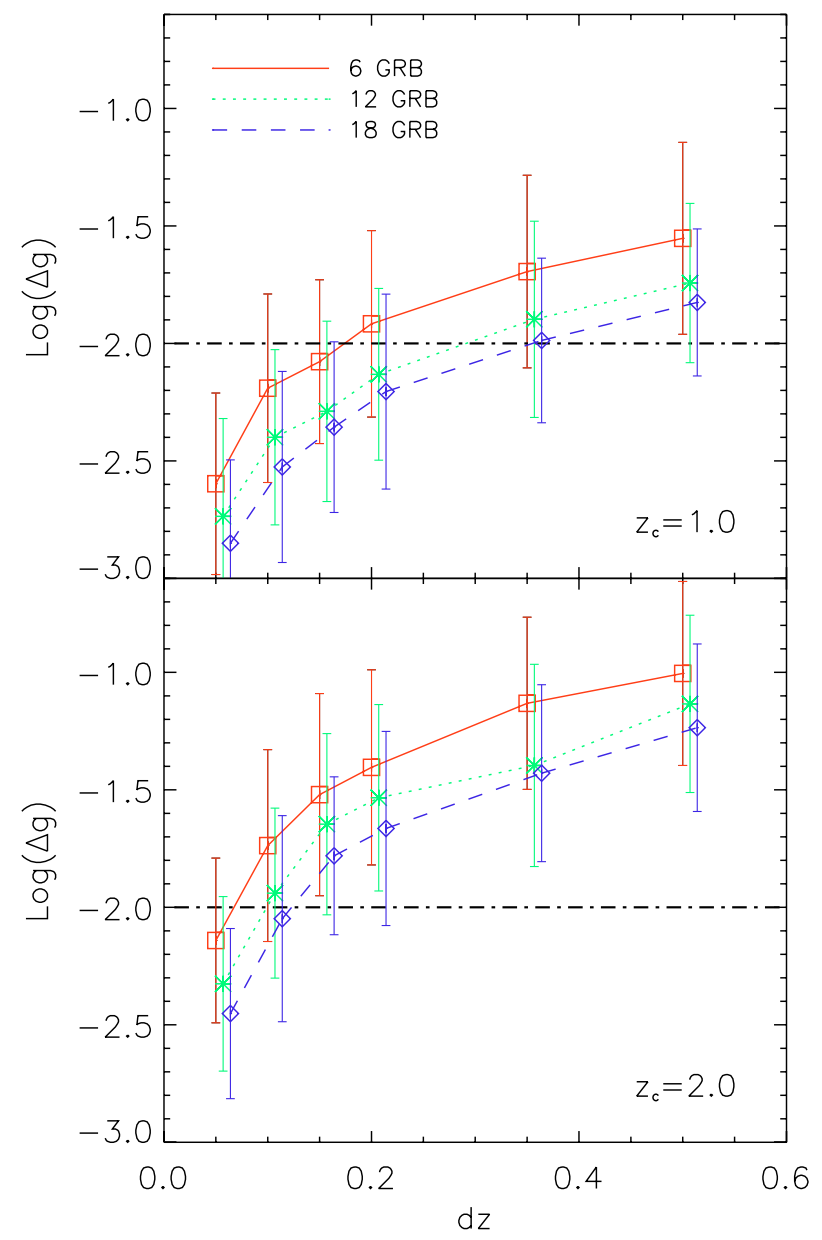

Fig. 8. Calibration of the $E_{\text {peak }}-E_{\gamma}^{g}$ correlation. For different samples of GRBs $(6,12,18$ - corresponding to the solid, dotted and dashed lines respectively) we show the maximum variation $\Delta g$ of the slope of the correlation for any cosmology $\Omega \in(0,1.5)$ as a function of the redshift dispersion of the GRBs $\mathrm{d} z$. The dot dashed line represents the limit of variation of $1 \%$ of the slope of the correlation. Data points have been shifted along the abscissa for graphycal purposes. 
In principle this issue could be solved (a) with a considerably large number of calibrators, i.e. low redshift GRBs for which the luminosity distance $d_{\mathrm{L}}$ is practically independent from the cosmological parameters, or (b) with a convincing theoretical interpretation of the physical nature of this correlation. In both cases the slope of the correlation would be fixed.

Case (a) could be realized with 5-6 GRBs at $z<0.1$. However, if (long) GRBs are produced by the core-collapse of massive stars, their rate is mainly regulated by the cosmic SFR and, therefore, the probability of detecting events at $z<0.1$ is small $\left(\sim 2 \times 10^{-5}\right)$. This number should be convolved with the GRB luminosity function: with the assumptions (described in Sect. 4) of our simulation we estimate that $\sim 1.3 \%$ of the 150 GRBs should be at low redshifts (i.e. $z<0.4$ ). Instead we should expect to have more chances to detect a considerable number (up to $\sim 31 \%$ ) of intermediate redshift GRBs $(z \sim 1-2)$ where the cosmic SFR peaks.

For this reason we explore the possibility to calibrate the correlation using a sufficient number of GRBs within a small redshift bin centered around any redshift. In fact, if we could have a sample of GRBs all at the same redshift the slope of the Ghirlanda correlation would be cosmology independent. Our objective is, therefore, to estimate the minimum number of GRBS $N$ within a redshift bin $\mathrm{d} z$ centered around a certain redshift $z_{\mathrm{c}}$ which are required to calibrate the correlation.

In practice the method consists in fitting the correlation for every choice of $\Omega$ using a set of $N$ GRBs distributed in the interval $\mathrm{d} z$ (centered around $z_{\mathrm{c}}$ ). We consider the correlation to be calibrated (i.e. its slope to be cosmology independent) if the change of the slope $g$ is less than $1 \%$.

The free parameters of this test are the number of GRBs $N$, the "redshift slice" $\mathrm{d} z$ and the central value of the redshift distribution $z_{\mathrm{c}}$. By Monte Carlo technique we use the same sample simulated in Sect. 4 under the WM assumption to minimize the variation of $\Delta g\left(\Omega ; N, \mathrm{~d} z, z_{\mathrm{c}}\right)$ over the $\Omega_{\mathrm{M}}, \Omega_{\Lambda} \in(0,1.5)$ plane as a function of the free parameters $\left(N, \mathrm{~d} z, z_{\mathrm{c}}\right)$.

We tested different values of $z_{\mathrm{c}}$ and different redshift dispersions $\mathrm{d} z \in(0.05,0.5)$. We required a minimum number of 6 GRBs to fit the correlation in order to have at least 4 degrees of freedom. We report our results in Fig. 8. We show the variation of $\Delta g$ as a function of $\mathrm{d} z$ for different samples $(6,12$, 18 GRBs - solid, dotted and dashed curves in Fig. 8). The error bars show the width of the distribution of the simulation results and not the uncertainty on the average value. At any redshift the fewer the number $N$ of GRBs the larger the change of $\Delta g$ (for the same $\mathrm{d} z$ ) because the correlation is less constrained. The dependence from $z_{\mathrm{c}}$ is instead different: for larger $z_{\mathrm{c}}$ we require a smaller bin $\mathrm{d} z$ to keep $\Delta g$ small.

From the curves reported in Fig. 8 we can conclude that already 12 GRBs with $z \in(0.9,1.1)$ might be used to calibrate the slope of the $E_{\text {peak }}-E_{\gamma}$ correlation. At redshift $z_{\mathrm{c}}=2$ instead we require a smaller redshift bin i.e. $z \in(1.95,1.05)$. We find that $N=12$ GRBs with $z \in(0.45,0.75)$ can be used to achieve the same $1 \%$ precision in the calibration. However, one key ingredient is that the GRBs used to calibrate the correlation do not have the same peak energy otherwise they would collapse in one point in the $E_{\text {peak }}-E_{\gamma}$ plane. Within the present sample of 19 GRBs there are only 4 GRBs within the redshift interval $0.4-0.8$ (i.e. $050525,041006,020405$ and 051022$)$ and 2 of these $(050525$ and 041006 ) have a very similar $E_{\text {peak }}$.

\section{Conclusions}

We have presented the cosmological constraints that can be obtained with the present sample of 19 GRBs for which all the relevant quantities, i.e. redshift $z$, peak energy $E_{\text {peak }}$ and jet break time $t_{\mathrm{b}}$, has been properly estimated and published in the literature.

Following the results of N05, where the $E_{\text {peak }}-E_{\gamma}$ correlation found under the hypothesis of a uniform jet model with either a homogeneous (HM) or a wind density profile (WM) were presented, we derived the constraints on $\Omega_{M}$ and $\Omega_{\Lambda}$ in these two scenarios.

We also presented the future of GRBs as cosmological tools. By simulating a sample of 150 GRBs (which is comparable in number to the present sample of "Gold" SN Ia) we showed that similar (to SN Ia) tight constraints can be obtained with GRBs either on the present universe composition and on the nature and evolution of DE (parametrized with $w_{0}$ and $w_{\mathrm{a}}$ ). We remark that the collection of such a large sample of GRBs which can be used as standard candles requires an accurate measurement of their prompt and afterglow properties. In particular a wide energy spectral coverage is required to constrain the peak energy and properly compute the bolometric corrected GRB energetics.

Finally, a large sample of GRBs would help in calibrating the slope of the $E_{\text {peak }}-E_{\gamma}$ correlation (in both the HM or WM case). To this aim one would require to collect enough low redshift GRBs. However, we can relax this requirement: we showed that even if not concentrated at very low $z$, it is sufficient to have a dozen GRBs with a similar redshift to find the slope of the correlation in a cosmology-independent way at the level of $1 \%$ accuracy.

Acknowledgements. We thank A. Celotti and V. Avila-Reese for useful discussions. We thank the Italian MIUR and INAF for founding (Cofin grant 2003020775_002).

\section{References}

Amati, L., Frontera, F., Tavani, M., et al. 2002, A\&A, 390, 81

Band, D., Matteson, J., Ford, L., et al. 1993, ApJ, 413, 281

Butler, N. R., Ricker, G. R., Lamb, D. Q., et al. 2005, GCN, 4165

Chevalier, R. A., \& Li, Z. Y. 2000, ApJ, 536, 195

Firmani, C., Ghisellini, G., Ghirlanda, G., \& Avila-Reese, V. 2005, MNRAS, 360, L1

Ghirlanda, G., Ghisellini, G., Lazzati, D., \& Firmani, C. 2004a, ApJ, 613, L13

Ghirlanda, G., Ghisellini, G., \& Lazzati, D. 2004b, ApJ, 616, 331

Ghisellini, G., Ghirlanda, G., Firmani, C., Lazzati, D., \& Avila-Reese, V. 2005, Il Nouvo Cimento, in press

Golenetskii, S., Aptekar, R., Mazets, E., et al. 2005, GCN, 4150

Kawai, N., Yamada, T., Kosugi, G., Hattori, T., \& Aoki, K. 2005, GCN Circ, 3937

Lamb, D., Ricker, G. R., Lazzati, D., et al. 2005 [arXiv: astro-ph/0507362]

Liang, E., \& Zhang, B. 2005, ApJ, in press [arXiv: astro-ph/0504404]

Linder, E. V., \& Huterer, D. 2005, Phys. Rev. D, 72, 043509

Nava, L., Ghisellini, G., Ghirlanda, G., Tavecchio, F., \& Firmani, C. 2006, A\&A, 450,471

Olive, F., et al. 2005, GCN, 4131

Porciani, C., \& Madau, P. 2001, ApJ, 548, 522

Racusin Kennea, J., Fox, D., et al. 2005, GCN, 4169

Riess, A. G., Strolger, L.-G., Tonry, J., et al. 2004, ApJ, 607, 665

Sari, R. 1999, ApJ, 524, L43

Xu, D., Dai, Z. G., \& Liang, E. W. 2005, ApJ, in press

[arXiv:astro-ph/0501458] 